\title{
HUMAN CAPITAL AND PREDATION: A POSITIVE THEORY OF EDUCATIONAL POLICY
}

\author{
Herschel I. Grossman \\ Minseong Kim
}

Working Paper 6403 
NBER WORKING PAPER SERIES

\title{
HUMAN CAPITAL AND PREDATION: A POSITIVE THEORY OF EDUCATIONAL POLICY
}

\author{
Herschel I. Grossman \\ Minseong Kim
}

Working Paper 6403

http://www.nber.org/papers/w6403

\section{NATIONAL BUREAU OF ECONOMIC RESEARCH \\ 1050 Massachusetts Avenue \\ Cambridge, MA 02138 \\ February 1998}

We acknowledge helpful comments from Juan Mendoza and Suk Jae Noh. We also appreciate the comments from the seminar participants at the Macroeconomic Workshop of the Korea Econometric Society, at the Midwest Macroeconomics Conference, at Hong Kong University of Science and Technology, and at Instituto Technologico Autonomo de Mexico. Any opinions expressed are those of the authors and not those of the National Bureau of Economic Research.

(C) 1998 by Herschel I. Grossman and Minseong Kim. All rights reserved. Short sections of text, not to exceed two paragraphs, may be quoted without explicit permission provided that full credit, including (C) notice, is given to the source. 
Human Capital and Predation: A Positive Theory

of Educational Policy

Herschel I. Grossman and Minseong Kim

NBER Working Paper No. 6403

February 1998

JEL Nos. D31, D74, H40, I28

\section{$\underline{\text { ABSTRACT }}$}

This paper offers an explanation for observed differences across countries in educational policies and in resulting interpersonal distributions of human capital. We analyze a generalequilibrium model in which, as a result of the apportionment of natural ability, nurturing, and publicly financed education, some people can be well endowed with human capital, whereas other people are poorly endowed with human capital. We assume that people can choose to be either producers or predators. An increase in a person's human capital makes predation a less attractive choice for that person. As a result, it is possible that by using some of their human capital to educate the poorly endowed people rather than to produce consumables the well endowed people can increase their own consumption. We also find that the nature of the educational policy that maximizes the consumption of the well endowed people depends on the ability of producers to enforce a collective choice of the amount of resources to be allocated to guarding against predators. Our theory predicts that, if producers collectively choose the amount of guarding against predators, then the well endowed people prefer a relatively egalitarian educational policy that increases the human capital of all of the poorly endowed people. Such an educational policy either decreases the cost of deterring predation or makes deterrence possible. In contrast, if producers or small subsets of producers individually choose the amount of their resources to allocate to guarding, taking the ratio of predators to producers as given, then the well endowed people prefer a more elitist educational policy that decreases the number of poorly endowed people, thereby decreasing the number of predators, without increasing the human capital of the remaining poorly endowed people. These implications seem to be consistent with the facts about differences across countries in educational policy.

Herschel I. Grossman

Department of Economics

Brown University

Box B

Providence, RI 02912

and NBER

herschel@brownvm.brown.edu
Minseong Kim

Department of Economics

University of Pittsburgh

Pittsburgh, PA 15260 
Mr. Benson looks to the future and sees a widening social abyss. "We could get to a situation similar to Manila, where the upper and middle classes surround themselves with walls and security guards to protect themselves from an abandoned underclass." ... When asked about Mr. Benson's fear of an urban America that looks like Manila, Mr. Newsome shrugs: "It's already like that," he says. (The Wall Street Journal, September 26, 1995, page A8.)

Although almost every modern state provides either public education or public subsidies for education, casual observation reveals that differences in educational policy result in large differences across countries in the interpersonal distribution of human capital. Most notably, in Western European countries and in some East Asian countries, like Japan, Korea, Singapore, and Taiwan, every person receives a good enough education to insure that almost everyone is at least functionally literate. In these countries the resulting distribution of human capital is relatively egalitarian. In contrast, in many of the countries of South America, Africa, and Asia, including the Philippines, and, as Mr. Newsome suggests, also in the United States, educational policy is more elitist. In these countries, while many people receive higher education and acquire a large amount of human capital, many other people receive little or no effective education and do not even achieve functional literacy. The distribution of human capital in these countries is highly unegalitarian.

This paper proposes a positive theory of the redistributional component of publicly financed education. This theory suggests both an explanation for the size of this redistributional component as well as an explanation for observed differences across countries in educational policies and in resulting interpersonal distributions of human capital.

Our analytical framework is a general-equilibrium model in which, as a result of the apportionment of natural ability, nurturing, and publicly financed education, some people can be well endowed with human capital, whereas other people are poorly endowed with human capital. In this model the interpersonal distribution of human capital has two dimensions. 
One dimension is the endowment of human capital of a poorly endowed person relative to a well endowed person. The other dimension is the fraction of people who are poorly endowed.

In theorizing about educational policy we focus on the predatory threat posed by "an abandoned underclass" to which Mr. Benson refers. To analyse this predatory threat we model people as choosing to be either producers or predators. Predators are people who produce nothing, but live by appropriating the product of the producers. We assume that each person chooses to be either a producer or a predator depending on whether production or predation yields more consumption for him. We also assume that the technologies of production and predation are such that the consumption that a person can obtain from being a producer is an increasing function of his human capital, whereas the consumption that a person can obtain from being a predator does not depend on his human capital. ${ }^{1}$

The possibility that some people might choose to be predators causes producers to allocate resources to guarding against predators. ${ }^{2}$ Guarding against predators includes all actions that are costly but have the effect of decreasing the fraction of production lost to predation. Examples of ways of guarding against predators include the locating of production in inconvenient but secure places, the production of things that are harder for predators to appropriate, the installation of locks, the building of walls, the hiring of private security guards, and the organizing of a police force. For simplicity, our analysis focuses only the

\footnotetext{
${ }^{1}$ This assumption implies that our analysis is applicable to low-skilled predatory activities like burglary, robbery, and kidnapping. We implicitly abstract from high-skilled predatory activities like embezzlement and some forms of litigation.

${ }^{2}$ Usher (1987) developed a pioneering general-equilibrium model in which people decide whether to be producers or predators and in which producers also decide how much time and effort to put into guarding against predation. In another paper - Grossman and Kim (1997a) - we analyse the choices of people to be producers or predators in a model in which only some people, whom we define to be amoral, are potential predators. The other people, whom we define to be moral, always choose to be producers, no matter how lucrative predation is relative to production. In the present paper we implicitly assume that everybody is amoral. Hence, every person is a potential predator.
} 
total amount of resources allocated to guarding, abstracting from different ways of guarding.

The existence of poorly endowed people who might choose to be predators negatively affects the consumption of well endowed people both because producers allocate some of their potentially productive resources to guarding against predators and because, unless predation is deterred, producers lose some of their production to predation. The threat of predation connects to educational policy because, as our analysis shows, the well endowed people can mitigate these effects by using some of their human capital to educate people who, because of either modest natural ability or ineffective nurturing or both, are initially poorly endowed with human capital. ${ }^{3}$ The educational policy that the well endowed people collectively choose can be either egalitarian, in that it increases the human capital of all of the poorly endowed people, or elitist, in that it only decreases the number of poorly endowed people without increasing the human capital of the remaining poorly endowed people, or some combination of egalitarian and elitist.

Our theory emphasizes that a decision to allocate resources to guarding against predators can be made either individually - that is, by single producers or by small subsets of producers - or collectively - that is, by a coordinated decision of all of the producers. An irreversible collective choice of the amount of guarding incorporates the strategic advantage

\footnotetext{
${ }^{3}$ We implicitly assume that the cost of receiving a publicly financed education, which would include the opportunity cost of time spent in school, is small enough that an initially poorly endowed person would accept any offer of publicly financed education. Previous papers, Grossman $(1994,1995)$, considered the possibility that the optimal response of a propertied class to the threat of predation could be a redistribution of either property or the income from property. In contrast to the present paper, in these papers the prey was endowed with physical property rather than with human capital, and the potential predators were also suppliers of labor that cooperated with physical capital in production. But, all of these papers have in common the derivation of a policy that maximizes the consumption of the people with the largest consumption. In Grossman and Kim (1997b) we use a model similar to the model in the present paper to derive the distribution of human capital that maximizes the consumption of the person with the lowest consumption.
} 
of taking into account the deterrent effect of guarding on the number of people who choose to be predators. But, in any society the enforcement of collective choices is problematic. Our theory focuses specifically on the limited ability of producers to enforce a collective choice of the amount of guarding. ${ }^{4}$ We consider three possible cases.

In the first case, the limited ability of producers to enforce a collective choice of the amount of guarding against predators does not impose a binding constraint. In the second case, the limited ability of producers to enforce a collective choice of the amount of guarding imposes a binding constraint, but with an appropriate redistributive educational policy this constraint is not tight enough to negate the strategic advantage of collective choice. In the third case, the producers' ability to enforce a collective choice of the amount of guarding is so limited that the strategic advantage of collective choice is lost for any feasible redistributive educational policy.

In this third case at the margin producers (or small subsets of producers) individually choose the amount of resources to allocate to guarding. Importantly, an individual producer takes both the amount of guarding chosen by other producers and the choices of other people to be either predators or producers as given. In other words, an individual producer (or small subset of producers) ignores the deterrent effect of guarding on the number of people who choose to be predators. Casual observation suggests that Western European countries and some East Asian countries, like Japan, Korea, and Singapore, and Taiwan, provide historical examples in which producers collectively choose the amount of guarding against predators, whereas other countries, mainly in South America, Africa, and Asia, including the Philippines and, perhaps, also the United States, provide historical examples in which

\footnotetext{
${ }^{4}$ To be more concrete, we can think of government as being the agent to whom producers assign the task of enforcing a collective choice of the amount of guarding, with taxation being the means of enforcement. In this context limited ability to enforce an irreversible collective choice of the amount of guarding reflects limited ability either to collect taxes or to allocate them for this purpose.
} 
at the margin producers individually choose the amount of guarding. ${ }^{5}$

Our analysis implies, as we shall see, that the benefits to well endowed people from either an egalitarian educational policy or an elitist educational policy depend on whether at the margin producers choose the amount of guarding collectively or individually. Specifically, we find that, in the first two cases in which producers collectively choose the amount of guarding against predators the well endowed people prefer an educational policy that increases the human capital of all of the poorly endowed people. Such an educational policy either decreases the cost of deterring predation or makes deterrence possible. In contrast, in the third case, in which at the margin producers individually choose the amount of their resources to allocate to guarding, taking the ratio of predators to producers as given, the well endowed people prefer an educational policy that decreases the number of poorly endowed people, thereby decreasing the number of predators, without increasing the human capital of the remaining poorly endowed people.

This analysis leads us to expect those countries in which producers collectively choose the amount of guarding to have egalitarian educational policies that provide even the poorly endowed with a relatively good education. In contrast, the analysis leads us to expect those countries in which at the margin producers individually choose the amount of guarding to have elitist educational policies that give some people an excellent education and other people little or no education. We motivate study of our model by observing that these implications seem to be consistent with observed differences across countries in educational policy.

\footnotetext{
${ }^{5}$ The limited ability of producers to enforce a collective choice of the amount of guarding can impose a tighter constraint in some countries than in others either because producers in the latter countries if unconstrained would choose a smaller amount of guarding or because producers in the latter countries are able to enforce a collective choice of a larger amount of guarding. In this paper we do not attempt to explain differences among countries in the ability of producers to enforce collective choices. Related papers by William Easterly and Ross Levine (1997) and Alberto Alesina, Reza Baqir, and Easterly (1997) suggest that difficulties in providing public goods are attributable to political polarization caused by ethnic diversity.
} 


\section{Analytical Framework}

Let $K$ denote the human capital of each well endowed person, and let $k$ denote the human capital of each poorly endowed person, where $K \geq k>0$. Also, let $u$ denote the nonnegative fraction of people who are poorly endowed, and let $U \equiv \frac{u}{1-u}$ denote the ratio of poorly endowed people to well endowed people. Aside from their endowments of human capital, people are otherwise identical. The average endowment of human capital, denoted by $\Omega$, is

$$
\Omega=(1-u) K+u k \equiv \frac{K+U k}{1+U} .
$$

Let $e$, where $0 \leq e<1$, denote the fraction of his human capital that each well endowed person allocates to educating people who are initially poorly endowed with human capital. The variable $e$ captures the redistributional component of publicly financed education. Assuming that each unit of human capital allocated to education produces one additional unit of human capital, $\Omega$ is related to the average initial endowment of human capital, denoted by $\Omega_{o}$, according to

$$
\Omega=\Omega_{o}+(1-u) e K \equiv \Omega_{0}+\frac{e K}{1+U}
$$

Equating these two expressions for $\Omega$ and rearranging, we can relate $(1-e) K$, the amount of human capital that each well endowed person has available for the production of consumables, to $\Omega_{o}, U$, and $k$. Specifically,

$$
(1-e) K=\Omega_{o}+U\left(\Omega_{o}-k\right)
$$

Equation (1) implies that, for a given value of $\Omega_{o}$, the combination of $U$ and $k$ fully describes the interpersonal distribution of human capital available for the production of consumables. ${ }^{6}$

\footnotetext{
${ }^{6}$ If each unit of human capital allocated to education produces $\lambda$ additional units of human capital, where $\lambda$ is positive but not necessarily equal to one, then equation (1) generalizes to $(1-\lambda e) K=\Omega_{0}+U\left(\Omega_{0}-k\right)$. All of the qualitative conclusions derived below obtain whether or not $\lambda$ equals one.
} 
Given the amount of human capital that he has available for production each person has to make two choices. First, he must choose whether to be a producer or a predator. Each person makes this choice individually, taking as given his potential consumption as a producer or as a predator. Let $N$ denote the nonnegative fraction of people who are well endowed and who choose to be producers, where $N \leq 1-u$, let $n$ denote the nonnegative fraction of people who are poorly endowed and who choose to be producers, where $n \leq u$, and let $r$ denote the nonnegative fraction of people, whether well endowed or poorly endowed, who choose to be predators. Let $R \equiv \frac{r}{N+n} \equiv \frac{r}{1-r}$ denote the ratio of predators to producers.

Second, if a person chooses to be a producer, then he must decide how to allocate his human capital between production and guarding against predators. As discussed above, at the margin this choice can be made either collectively or individually. Let $g$ denote the nonnegative fraction of the human capital that a producer has available for production that he allocates instead to guarding against predators, and let $G \equiv \frac{g}{1-g}$ denote the ratio of the human capital that a producer allocates to guarding against predators to the human capital that he allocates to the production of consumables. Assume that $\bar{G}$ is the maximum irreversible collective choice of $G$ that producers can enforce.

To simplify the analysis of the choice between being a producer or a predator, assume that a unit of human capital can produce one unit of consumables. The number of units of consumables that a person actually produces equals the product of the amount of human capital that he has available for production and the fraction of this human capital that he allocates to production. ${ }^{7}$

\footnotetext{
${ }^{7}$ In this setup individual productive activities are independent. We also abstract from trade in either productive inputs or consumables and from any externalities. François Bourguignon and Thierry Verdier (1996) analyze a model in which positive externalities can induce well endowed people to subsidize the education of poorly endowed people. In the present model, well endowed people have an incentive to educate poorly endowed people only to mitigate the predatory threat from the poorly endowed people.
} 
A producer appropriates the nonnegative fraction $p$ of his production, and predators appropriate the nonnegative fraction $1-p$. Assume that $p$ depends negatively on the ratio of predators to producers, $R$, and positively on $G$. Specifically,

$$
p=\frac{1}{1+\theta R / G}, \quad \theta \geq 0
$$

In equation (2), the parameter $\theta$, which embodies the technology of predation, determines the effectiveness of predators in appropriating consumables for given values of $R$ and $G$. The specification that $p$ depends on the number of predators but not on the identity of the predators reflects the assumption that well endowed people and poorly endowed people are equally effective at predation. ${ }^{8}$

Let $C$ denote the consumption of a well endowed producer and let $c$ denote the consumption of a poorly endowed producer. After allowing for the fraction of human capital that well endowed people allocate to education, for the fraction of human capital that producers allocate to guarding against predators, and for the fraction of consumables lost to -predators, we have

$$
C=p(1-g)(1-e) K
$$

and

$$
c=p(1-g) k
$$

\footnotetext{
${ }^{8}$ Equation (2) is a generic black box that conceals the process of predation, just as the standard generic production function conceals the process of production. For example, the relation between appropriative inputs and the appropriative outcome described by equation (2) could involve either the use of force or a peaceful settlement under the threat of force, although, strictly speaking, given complete information and the absence of stochastic factors, this model does not provide an internal explanation for costly violence. Dagobert Brito and Michael Intriligator (1985) address the question of whether appropriative conflict is resolved with or without violence and destruction, and emphasize the importance of incomplete information as a cause of violence.
} 
Finally, let $D$ denote the consumption of a predator. ${ }^{9}$ Assuming that each predator obtains an equal share of the total amount of consumables appropriated from the producers, we have

$$
D=\frac{(1-p)(1-g)[N(1-e) K+n k]}{r}
$$

According to equation (5) $D$ is a decreasing function of $r$ and is well defined for positive values of $r$. Further, if $r$ equals zero, which implies that $N$ equals $1-u$ and that $n$ equals $u$, then the value of $D$ is defined to be $\lim _{r \rightarrow 0} D$, which, using equations (1) and (2), equals $\frac{\theta}{G}(1-g) \Omega_{o}$.

\section{The Choice to be a Producer or a Predator}

To decide whether to be a producer or a predator, each well endowed person compares the values of $C$ and $D$, which he takes as given, and each poorly endowed person compares the values of $c$ and $D$, which he takes as given. Using equations (1) - (5) we find that the choices of well endowed and poorly endowed people to be producers or predators depend on $G$ in the following way:

1. If $D$ were larger than both $c$ and $C$, then every person would prefer to be a predator. This case could occur if and only if $G$ were smaller than $\theta$. In this case, $R$ would be infinite.

2. If $D$ equals $C$ but is larger than $c$, then poorly endowed people prefer to be predators, whereas well endowed people are indifferent between being producers or predators. This case would occur if and only if $G$ equals $\theta$. In this case, $R$ can take any value larger than or

\footnotetext{
${ }^{9}$ For simplicity, the model assumes that predators only prey on producers. Predators do not prey on other predators. The model also abstracts from possible destruction of a some consumables as the result of predation. In Grossman and Kim (1995) we show how destruction is easily incorporated into the analysis. In addition, we could modify the model to allow the activity of guarding against predators to include the apprehension and punishment of predators. The apprehension and punishment of predators would not directly affect $p$, but would reduce the expected utility of predators.
} 
equal to $U$.

3. If $D$ is smaller than $C$ but larger than $c$, then poorly endowed people prefer to be predators, whereas well endowed people prefer to be producers. This case would occur only if $G$ is larger than $\theta$, but smaller than $\theta(1-e) K / k$. In this case, $R$ is equal to $U$.

4. If $D$ is smaller than $C$ but equal to $c$, then poorly endowed people are indifferent between being producers or predators, whereas well endowed people prefer to be producers. This case would occur only if $G$ is equal to or larger than $\theta \Omega_{o} / k$, but not larger than $\theta(1-e) K / k$. In this case, the equality between $D$ and $c$ implies that $R$ is equal to $\frac{G-\theta \Omega_{0} / k}{\theta\left(\frac{\Omega_{2}}{k}-1\right)}$. This implied value of $R$ is equal to or smaller than $U$, but larger than or equal to zero.

5. If $D$ were smaller than both $c$ and $C$, then every person would prefer to be a producer. This case could occur only if $G$ were larger than $\theta \Omega_{o} / k$. In this case, $R$ would be zero. Summarizing these results, the choices of well endowed and less endowed people to be producers or predators are such that

$$
R=\left\{\begin{array}{lrl}
\infty & \text { if and only if } & G<\theta \\
x \in[U, \infty] & \text { if and only if } & G=\theta \\
U & \text { only if } & \theta<G<\theta(1-e) K / k \\
\frac{G-\theta \Omega_{o} / k}{\theta\left(\frac{\Omega_{0}}{k}-1\right)} & \text { only if } & \theta \Omega_{o} / k \leq G \leq \theta(1-e) K / k \\
0 & \text { only if } & G>\theta \Omega_{o} / k
\end{array}\right.
$$

\section{The Ratio of Predators to Producers with Unconstrained Collective Choice of the Amount of Guarding}

Consider a country in which the limited ability of producers to enforce an irreversible collective choice of the amount of guarding against predators, as represented by $\bar{G}$, does not impose a binding constraint. In this country the producers collectively choose $G$ to -maximize $C$ and $c$, as given by equations (3) and (4). This unconstrained collective choice of $G$ takes into account both the effect of $G$ on $p$ for a given ratio of predators 
to producers, as given by equation (2), and the effect of $G$ on the choices of well endowed and poorly endowed people to be predators, as given by equation (6). ${ }^{10}$ Because a producer choosing his amount of guarding individually would not take into account the effect of $G$ on $R$, the unconstrained collective choice of $G$ is as large as or larger than producers would choose individually. Hence, given an unconstrained collective choice of $G$ producers would not individually choose to allocate any additional resources to guarding.

Substituting equations (2) and (6) into equations (3) and (4), we find that both $C$ and $c$ have a local maximum at $G=(1+\epsilon) \theta(1-e) K / k$, where $\epsilon$, a small positive number, is the smallest fraction by which the producers collectively can choose to increase $G$. From equation (6), $(1+\epsilon) \theta(1-e) K / k$ is the minimum value of $G$ that would result in an equilibrium in which $R$ uniquely equals zero - that is, the minimum value of $G$ that would surely deter every person, whether well endowed or poorly endowed, from choosing to be a predator. ${ }^{11}$ We also find that both $C$ and $c$ can have either one of two other local maxima. One of these possible local maxima is at $G=\sqrt{\theta U}$, which is the solution to the first-order conditions for an interior maximum, $d C / d g=d c / d g=0$, given $R=U$. The other possible local maximum is at $G=(1+\epsilon) \theta$, which is the minimum value of $G$ that would deter the well endowed people, but not the poorly endowed people, from choosing to be predators.

The existence of these possible local maxima depends on the values of $k$ and $U$ in the

\footnotetext{
${ }^{10}$ Because the model assumes that for each producer $p$ depends only on $R$ and on his own amount of guarding, the potential gain from collective choice of the amount of guarding involves only the strategic advantage from taking into account the effect of $G$ on $R$. If $p$ also depended either positively or negatively on the amount of resources that other producers allocate to guarding, then collective choice of the amount of guarding also would allow producers to take these technological externalities into account. Another possible complication from which we abstract is that some ways of guarding that can only be chosen collectively could be more efficient than other ways of guarding.

${ }^{11}$ Equation (6) says that $R=0$ could also be associated with values of $G$ such that $\theta(1-e) K / k \geq$ $G>\theta \Omega / k$. But, such values of $G$ also can be associated with positive values of $R$.
} 
following way:

1. If $(1-e) K / k \geq \sqrt{U / \theta}>1$, then $G=(1+\epsilon) \theta(1-e) K / k$ and $G=\sqrt{\theta U}$ are both local maxima.

2. If $(1-e) K / k>1 \geq \sqrt{U / \theta}$, then $G=(1+\epsilon) \theta(1-e) K / k$ and $G=(1+\epsilon) \theta$ are both local maxima.

3. If $\sqrt{U / \theta}>(1-e) K / k \geq 1$, then $G=(1+\epsilon) \theta(1-e) K / k$ is the unique local maximum. To determine the global maximum, we compare the values of $C$ and $c$ associated with $G$ equal to $(1+\epsilon) \theta(1-e) K / k, \sqrt{\theta U}$, and $(1+\epsilon) \theta$. The values of $C$ that would result from each of these possible choices of $G$ are

$$
C= \begin{cases}\frac{(1-e) K}{1+(1+\epsilon) \theta(1-e) K / k} & \text { for } G=(1+\epsilon) \theta(1-e) K / k \\ \frac{(1-e) K}{(1+\sqrt{\theta U})^{2}} & \text { for } G=\sqrt{\theta U} \\ \frac{(1-e) K}{\left(1+\frac{U}{1+\epsilon}\right)[1+(1+\epsilon) \theta]} & \text { for } G=(1+\epsilon) \theta\end{cases}
$$

The values of $c$ that would result from each of these possible choices of $G$ are the same, except that $k$ replaces $(1-e) K$ in the numerator of each expression. Given $k$ and $U$ the producers collectively choose the value of $G$ for which the resulting values of $C$ and $c$ are largest. Denote the resulting value of $C$ as $C_{J}$.

Equation (7) implies that, given $\Omega_{o}$, either the smaller is $(1-e) K / k$ or the larger is $U$ the more likely are the producers collectively to choose $G=(1+\epsilon) \theta(1-e) K / k$. The intuition for this result is that, the smaller is $(1-e) K / k$ the smaller is the amount of guarding that is needed to deter the poorly endowed people from choosing to be predators, whereas the larger is $U$ the larger would be the ratio of predators to producers and the resulting fraction of production lost to predation if the poorly endowed people were not deterred from choosing to be predators. 


\section{Educational Policy Given Unconstrained Collective Choice of the Amount of Guarding}

As discussed above, the well endowed people can make a collective choice of the fraction of their human capital to allocate to educating people who are initially poorly endowed with human capital. In addition, the educational policy that the well endowed people collectively choose can be either egalitarian, in that it increases the human capital of all of the poorly endowed people, or elitist, in that it only decreases the number of poorly endowed people, or some combination of egalitarian and elitist. The objective of the well endowed people is to maximize their own consumption.

The problem that the well endowed people solve is to choose $k$ and $U$ to maximize $C_{J}$, the consumption of a well endowed person, as derived from equation (7), subject to $k \geq k_{o}$ and $U \leq U_{o}$, where $k_{o}$ is the initial endowment of human capital of an initially poorly endowed person, and where $U_{o}$ is the ratio of initially poorly endowed people to initially well endowed people. Equation (1) implies that, given $\Omega_{o}$, the choice of $k$ and $U$ also determines $(1-e) K$, the amount of human capital that each well endowed person has available for the production of consumables. Assume further that $k_{o}$ is small - specifically, that $k_{o}$ is smaller than $(1-e) K \sqrt{\theta / U_{0}}$ - and that $U_{o}$ is larger than $\theta$.

Substituting equation (1) into equation (7) we find that, with $U$ larger than $\theta$, the maximum value of $C_{J}$ obtains for $G$ equal to $(1+\epsilon) \theta(1-e) K / k$ and $k /(1-e) K$ equal to $\sqrt{\theta / U}$. In addition, with $G$ equal to $(1+\epsilon) \theta(1-e) K / k$ and $k /(1-e) K$ equal to $\sqrt{\theta / U}$, the maximum value of $C_{J}$ is a nondecreasing function of $U$. Thus, with $k_{o} /(1-e) K$ smaller than $\sqrt{\theta / U_{0}}$, the solution to the problem of maximizing $C_{J}$ is for the initially well endowed people to leave $U$ unchanged and equal to $U_{o}$, but to increase $k$ to make

$$
k /(1-e) K=\sqrt{\theta / U_{o}} .
$$

Given this interpersonal distribution of human capital, the producers collectively choose

$$
G=(1+\epsilon) \theta(1-e) K / k=(1+\epsilon) \sqrt{\theta U_{o}} .
$$


From equation (9) we see that the assumption that $\bar{G}$ is not a binding constraint implies that $\bar{G}$ is at least as large as $(1+\epsilon) \sqrt{\theta U_{o}}$. (See Mathematical Appendix A for a more complete derivation of these results.)

This analysis says that, if the limited ability of producers to enforce a collective choice of the amount of guarding against predators does not impose a binding constraint, and given that $k_{o}$ is small and that $U_{o}$ is large, then the well endowed people choose an educational policy that is egalitarian in that it increases the human capital of all of the initially poorly endowed people. This educational policy, however, does not equalize everyone's human capital, nor does it decrease the fraction of people who are poorly endowed. Given the interpersonal distribution of human capital that results from this educational policy, the producers collectively chose enough guarding against predators to deter everyone from choosing to be a predator. ${ }^{12}$ In this case, the educational policy that the well endowed people choose balances the marginal benefit of decreasing the cost of deterring predation by increasing the human capital of poorly endowed people with the marginal cost of increasing the human capital of poorly endowed people.

\section{Educational Policy and The Ratio of Predators to Producers with Con- strained Collective Choice of the Amount of Guarding}

Consider another country in which the limited ability of producers to enforce an irreversible collective choice of the amount of guarding against predators, as represented by $\bar{G}$,

${ }^{12}$ This implication involves the implicit assumption that every person who is initially poorly endowed with human capital is sufficiently educable to be able to achieve the endowment $k$ as given by equation (8). In reality, it is likely that some people are not able to achieve this endowment of human capital. The choice of $G$ as given by equation (9) would not deter these uneducable people from choosing to be predators. Accordingly, we should not take the implication that everyone is deterred from predation literally. We should expect that in reality, even with unconstrained collective choice of the amount of guarding and a educational policy that attempts to increase everyone's human capital to $k$ as given by equation (8), some uneducable people choose to be predators. 
imposes a binding constraint. Specifically, assume that $\bar{G}$ is smaller than $(1+\epsilon) \sqrt{\theta U_{o}}$, but also assume that $\bar{G}$ is at least as large as $(1+\epsilon) \theta$. In other words, $\bar{G}$ is smaller than the choice of $G$ derived in the preceding section, but $\bar{G}$ is at least as large as the minimum value of $G$ that would deter the well endowed people from choosing to be predators.

In this country the well endowed people collectively choose the educational policy that maximizes $C$, given that the producers collectively choose $G$ to maximize $C$ and $c$, subject to the constraint $G \leq \bar{G} \cdot{ }^{13}$ Assume again that $k_{o}$ is smaller than $(1-e) K \sqrt{\theta / U_{0}}$ and that $U_{o}$ is larger than $\theta$.

Substituting equation (1) into equation (7) we find that, with $U$ larger than $\theta$, the maximum value of $C_{J}$, subject to the constraint $G \leq \bar{G}$, obtains for $k /(1-e) K$ equal to $(1+\epsilon) \theta / \bar{G}$ and $G$ equal to $\bar{G}$. In addition, with $k /(1-e) K$ equal to $(1+\epsilon) \theta / \bar{G}$ and $G$ equal to $\bar{G}$, the maximum value of $C_{J}$ is a nondecreasing function of $U$. Thus, with $k_{o} /(1-e) K$ smaller than $\sqrt{\theta / U_{o}}$, the solution to the problem of maximizing $C_{J}$ is for the well endowed people to leave $U$ unchanged and equal to $U_{o}$ and to increase $k$ to make

$$
k /(1-e) K=(1+\epsilon) \theta / \bar{G}
$$

Given this interpersonal distribution of human capital, the producers collectively choose

$$
G=(1+\epsilon) \theta(1-e) K / k=\bar{G}
$$

(See Mathematical Appendix B for a more complete derivation of these results.)

\footnotetext{
${ }^{13}$ This assumption implies that, although the limited ability of producers to enforce an irreversible collective choice of the amount of guarding against predators imposes a binding constraint, the well endowed people do not face a binding constraint on their ability to enforce the collective choice of an educational policy. A possible rationale for this assumption is that a collective choice of educational policy involves only the well endowed people, whereas a collective choice of the amount of guarding involves all of the producers. who can include poorly endowed people in addition to well endowed people.
} 
This analysis says that, given that the limited ability of producers to enforce an irreversible collective choice of the amount of guarding against predators imposes a binding constraint, but that producers can enforce a collective choice of guarding that is at least large enough to deter well endowed people from choosing to be predators, and given that $k_{0}$ is small and that $U_{0}$ is large, the well endowed people choose an egalitarian educational policy that increases the human capital of all of the initially poorly endowed people sufficiently to make deterrence possible. From equation (10) the assumption that $\bar{G}$ is smaller than $(1+\epsilon) \sqrt{\theta U_{o}}$ implies that $k /(1-e) K$ is larger than $\sqrt{\theta / U_{o}}$. Moreover, the smaller is $\bar{G}$ the larger that $k /(1-e) K$ has to be to make deterrence possible. In fact, if $\bar{G}$ equals $(1+\epsilon) \theta$, then $k /(1-e) K$ must equal one to make deterrence possible. Thus, in this case the educational policy that the well endowed people choose might come close to equalizing everyone's human capital.

Given the interpersonal distribution of human capital that results from this educational policy, the producers collectively chose the maximum amount of guarding against predators that they can enforce. This amount of guarding is enough to deter everyone from choosing to be a predator. Moreover, because this constrained collective choice of $G$ implies that predation is deterred, producers would not individually choose to allocate any additional resources to guarding.

\section{The Ratio of Predators to Producers with Individual Choice of the Amount of Guarding}

Finally, consider a country in which $\bar{G}$ is smaller than $(1+\epsilon) \theta$. In this country not only is $\bar{G}$ a binding constraint, but the maximum collective choice of $G$ that producers can enforce is smaller than the minimum value of $G$ that would deter the well endowed people from choosing to be predators. As a result, in this country, even if producers collectively choose the maximum amount of guarding that they can enforce, at the margin each producer would individually choose to allocate additional resources to guarding. 
In individually choosing the total amount of his resources to allocate to guarding, each producer takes the choices of other people to be producers or predators as given. Thus, if a well endowed person chooses to be a producer, then he chooses $G$ to maximize $C$, taking $R$ as given, and if a poorly endowed person were to choose to be a producer, then he would choose $G$ to maximize $c$, taking $R$ as given.

To analyse these choice problems we substitute equation (2) into equations (3) and (4) and calculate the value of the ratio $G$ that satisfies the conditions $d C / d g=0$ and $d c / d g=0$. For both well endowed producers and poorly endowed producers this ratio is given by

$$
G=\sqrt{\theta R}
$$

Solving equations (6) and (12) for $R$ and $G$, and assuming that the ratio $k / \Omega_{0}$ is small, we find that

$$
R=\max \{\theta, U\}
$$

and

$$
G=\max \{\theta, \sqrt{\theta U}\}
$$

Equation (13) says that, with individual choice of the amount of guarding, all of the poorly endowed people and, if the fraction of people who are poorly endowed is small, also some of the well endowed people choose to be predators. ${ }^{14}$ If $R$ equals $U$, then well

${ }^{14}$ More generally, solving equations (6) and (12) for $R$ would yield

$$
R= \begin{cases}\theta & \text { for } U \leq \theta \\ U & \text { for } \theta<U \leq R_{1} \\ R_{1} & \text { for } R_{1}<U<R_{2} \\ \left\{R_{1}, R_{2}, U\right\} & \text { for } U \geq R_{2},\end{cases}
$$

where $R_{1}$ and $R_{2}$ are the values of $R$ that satisfy both $R=\frac{G-\theta \Omega_{o} / k}{\theta\left(\frac{\Omega_{0}}{k}-1\right)}$, from equation $(6)$, and $G=\sqrt{\theta R}$, 
endowed people in equilibrium prefer to be producers, whereas if $R$ equals $\theta$, then well endowed people in equilibrium are indifferent between being producers and being predators. Equation (14) says that, with $\bar{G}$ smaller than $(1+\epsilon) \theta, \quad G$ is at least as large as $\bar{G}$, and that, if $U$ is larger than $\theta$, then $G$ is larger than $\bar{G}$.

Because well endowed people in equilibrium either prefer to be producers or are indifferent between being producers and being predators, the consumption of a well endowed person is equal to $C$. Substituting for $p$ from equation (2) and for $R$ and $G$ from equations (13) and (14) into equation (3), we calculate the equilibrium value of $C$, denoted $C_{I}$, to be

$$
C_{I}= \begin{cases}\frac{(1-e) K}{(1+\theta)^{2}} & \text { for } U \leq \theta \\ \frac{(1-e) K}{(1+\sqrt{\theta U})^{2}} & \text { for } U>\theta .\end{cases}
$$

\section{Educational Policy Given Individual Choice of the Amount of Guarding}

Assume again that the well endowed people make a collective choice of the fraction of their human capital to allocate to educating people who, because of either modest natural ability or ineffective nurturing or both, are initially poorly endowed with human capital. In this case the problem that the well endowed people solve is to choose $k$ and $U$ to maximize $C_{I}$, as given by equation (15), subject to $k \geq k_{o}$ and $U \leq U_{o}$. Also assume again that $U_{o}$ is larger than $\theta$.

Substituting equation (1) into equation (15) we find that $C_{I}$ is a decreasing function of $k$. Thus, part of the solution to the problem of maximizing $C_{I}$ is for the well endowed people to leave $k$ unchanged and equal to $k_{o}$. We also find that $C_{I}$ is an increasing function of $U$ for $U \leq \theta$ and that $C_{I}$ is a U-shaped function of $U$ for $U>\theta$. Specifically, $C_{I}$ has a local maximum at $U$ equal to $\theta$, and $C_{I}$ approaches a finite limit from equation (12). Such values of $R$ would exist only if $k / \Omega_{0}$ were sufficiently large. The larger is $\theta$ the larger $k / \Omega_{o}$ would have to be. 
from below as $U$ goes to infinity. If and only if $U$ equal to $\theta$ is not the global maximum, then there exists a value of $U$, denoted $\hat{U}$, such that $C_{I}$ evaluated at any value of $U$ larger than $\hat{U}$ is larger than $C_{I}$ evaluated at $U$ equal to $\theta$. (Note that $\hat{U}$, if it exists, is larger than $\theta$.)

This analysis implies that the other part of the solution to the problem of maximizing . $C_{I}$ may or may not call for the well endowed people to use some of their human capital to decrease $U$. If $\hat{U}$ exists and if $U_{o}$ is as large as or larger than $\hat{U}$, then the well endowed people leave $U$ unchanged and equal to $U_{o}$. In this case, the well endowed people do not choose to use any of their human capital to educate people who are initially poorly endowed because, with a small fraction of people who are initially well endowed, a given decrease in $U$ would require that a large fraction of the human capital of the well endowed people be used for education.

Alternatively, either if $\hat{U}$ does not exist or if $U_{o}$ is smaller than $\hat{U}$, then to maximize $C_{I}$ the well endowed people decrease $U$ to make $U$ equal $\theta$. In this case, the well endowed people choose to educate some of the initially poorly endowed people sufficiently to make them also well endowed, thereby decreasing the fraction of people who are poorly endowed, but leaving the remaining poorly endowed people with no more than their initial endowment of human capital. This elitist educational policy reduces $R$, the ratio of predators to producers, from $U_{o}$ to $\theta$. In other words, because of the producers' limited ability to enforce a collective choice of the amount of guarding, educational policy is directed towards decreasing the number of predators rather than facilitating the deterrence of predation.

\section{Summary}

Assuming that people can choose to be either producers or predators, we have found that the well endowed people can maximize their own consumption by choosing collectively to use some of their human capital to educate people who, because of modest natural ability or ineffective nurturing or both, are initially poorly endowed with human capital. More 
importantly, we found that whether the well endowed people choose an egalitarian educational policy that increases the human capital of all of the poorly endowed people or an elitist educational policy that only decreases the number of poorly endowed people without increasing the human capital of the remaining poorly endowed people depends on the ability of producers to enforce an irreversible collective choice of the amount of guarding against predators.

We considered three possible cases. In the first case, the limited ability of producers to enforce an irreversible collective choice of the amount of guarding does not impose a binding constraint. In the second case, the limited ability of producers to enforce a collective choice of the amount of guarding imposes a binding constraint, but this constraint is not tight enough to negate the strategic advantage of collective choice in taking into account the deterrent effect of guarding on the number of people who choose to be predators. In the third case, the producers' ability to enforce a collective choice of the amount of guarding is so limited that the strategic advantage of collective choice is lost.

We found that in the first two cases the well endowed people can maximize their own consumption by choosing an egalitarian educational policy that by increasing the human capital of all of the poorly endowed people either decreases the cost of deterring predation or makes deterrence possible. In contrast, in the third case at the margin producers or small subsets of producers individually choose the amount of resources to allocate to guarding. Importantly, an individual producer or small subset of producers takes both the amount of guarding chosen by other producers and the choices of other people to be either predators or producers as given, ignoring the deterrent effect of guarding on the number of people who choose to be predators. We found that in this case the well endowed people can maximize by their own consumption by choosing an elitist educational policy that decreases the number of poorly endowed people and, thereby, decreases the number of predators.

Applying this theory, we contrast two sets of countries. One set includes Western European countries and some East Asian countries, like Japan, Korea, Singapore, and Taiwan, 
that seem to provide historical examples of collective choice of the amount of guarding against predators, and in which, as a result, the amount of guarding has been sufficient to deter most, if not all, low-skilled predatory activities like burglary, robbery, and kidnapping. Our theory leads us to expect these countries to have egalitarian educational policies that provide even relatively poorly endowed people with a good education. The other set includes countries in South America, Africa, and Asia, including the Philippines, and, perhaps, also the United States, that seem to provide historical examples of individual choice of the amount of guarding against predators. Our theory leads us to expect these countries to have elitist educational policies that give some people an excellent education and other people little of no effective education. As we have noted, these implications seem to be consistent with the facts about differences across countries in educational policy. 


\section{Mathematical Appendices}

\section{A. Educational Policy Given Unconstrained Collective Choice of the Amount of}

\section{Guarding}

Let $C_{J}^{*}$ denote the global maximum of $C_{J}$ subject to the constraints $k_{o} \leq k \leq \Omega_{0}$ and $0 \leq U \leq U_{0}$. We find the values of $k$ and $U$ associated with $C_{J}^{*}$ by comparing the three possible local maxima, as given by equation (7), each maximized with respect to $U$ and $k$.

1. Substituting equation (1) into the first row of equation (7), we find that the local maximum for $C$ at $G=(1+\epsilon) \theta(1-e) K / k$ is maximized with $U=U_{o}$ and $k=\min \left\{\frac{\sqrt{1+\epsilon}\left(1+U_{o}\right) \Omega_{o}}{U_{o}+\sqrt{U_{o} / \theta}}, \Omega_{o}\right\}$, which implies $k /(1-e) K=\min \left\{\sqrt{\theta / U_{o}}, 1\right\}$. The resulting value of $C$, denoted $C^{I}$, is $C^{I}=\frac{\Omega_{o}\left(1+U_{o}\right)}{\left(1+\sqrt{\theta U_{o}}\right)\left[1+\sqrt{(1+\epsilon) \theta U_{o}}\right]}$ if $U_{o}>\theta$ and $C^{I}=\frac{\Omega_{0}}{1+(1+\epsilon) \theta}$ if $U_{0} \leq \theta$.

2. Substituting equation (1) into the second row of equation (7), we find that the local maximum of $C$ at $G=\sqrt{\theta U}$, if it exists, is maximized either with $U=(1+\epsilon) \theta$ and $k=k_{o}$ or with $U=U_{o}$ and $k=k_{0}$. The resulting value of $C$, denoted $C^{I I}$, is either $C^{I I}=\frac{[1+(1+\epsilon) \theta] \Omega_{o}-(1+\epsilon) \theta k_{o}}{(1+\theta \sqrt{1+\epsilon})^{2}}$ or $C^{I I}=\frac{\left(1+U_{o}\right) \Omega_{o}-U_{0} k_{o}}{\left(1+\sqrt{\theta U_{o}}\right)^{2}}$, respectively.

3. Substituting equation (1) into the third row of equation (7), we find that the local maximum of $C$ at $G=(1+\epsilon) \theta$, if it exists, is maximized with $U=0$. The resulting maximized value of $C$, denoted $C^{I I I}$, is $C^{I I I}=\frac{\Omega_{\supset}}{1+(1+\epsilon) \theta}$.

We can easily see that, if $U_{o}>\theta$, then $C^{I}>C^{I I}$ and $C^{I}>C^{I I I}$, regardless of whether $C^{I I}$ occurs with $U=(1+\epsilon) \theta$ or $U=U_{0}$. Thus, $C_{J}^{*}$ is associated with $U=U_{0}$ and $k$ and $G$ as given by equations (8) and (9).

B. Educational Policy With Constrained Collective Choice of the Amount of Guarding

Let $\bar{C}_{J}$ denote the global maximum of $C_{J}$ subject to the constraint $G \leq \bar{G}$ and subject to the constraints $k_{o} \leq k \leq \Omega_{o}$, and $0 \leq U \leq U_{0}$. Assume that $U_{0}>\theta$ and $(1+\epsilon) \theta \leq \bar{G}<(1+\epsilon) \sqrt{\theta U_{o}}$. Again, we find the values of $k$ and $U$ associated with $\bar{C}_{J}$ by 
comparing the three possible local maxima, as given by equation (7), each maximized with respect to $U$ and $k$.

1. Although the value of $G$ associated with the maximization of the first row of equation (7) with respect to $U$ and $k$ does not satisfy the constraint $G \leq \bar{G}$, by substituting equation (1) into $G=(1+\epsilon) \theta(1-e) K / k$ and rewriting the constraint $G \leq \bar{G}$ as $\frac{(1+\epsilon) \theta\left[\Omega_{0}+U\left(\Omega_{0}-k\right)\right]}{k} \leq \bar{G}$, we see that the binding constraint of $\bar{G}$ can be satisfied at $G=$ $(1+\epsilon) \theta(1-e) K / k$ either by decreasing $U$ or by increasing $k$. Because both decreasing $U$ and increasing $k$ reduces the value of $C$ at $G=(1+\epsilon) \theta(1-e) K / k$ from the unconstrained maximum, the constrained maximum would occur with the constraint $G \leq \bar{G}$ just satisfied. Substituting $G=\bar{G}=(1+\epsilon) \theta(1-e) K / k$ into the first row of equation (7), we find that, with the constraint $G \leq \bar{G}$ just satisfied, the local maximum for $C$ at $G=(1+\epsilon) \theta(1-\epsilon) K / k$ is $C=\frac{k \bar{G}}{(1+\epsilon) \theta(1+\bar{G})}$. This local maximum for $C$ is maximized with the largest value of $k$ that satisfies the constraints $U \leq U_{o}, k \leq \Omega_{o}$, and $G=\bar{G}$. Thus, this local maximum for $C$ is maximized with $U=U_{o}$ and $k=\frac{(1+\epsilon) \theta\left(1+U_{o}\right) \Omega_{o}}{(1+\epsilon) \theta U_{o}+\bar{G}}$, which implies $k /(1-e) K=(1+\epsilon) \theta / \bar{G}>\sqrt{\theta / U_{o}}$. The resulting value of $C$, denoted $\bar{C}^{I}$, is $\bar{C}^{I}=\frac{\bar{G}\left(1+U_{o}\right) \Omega_{o}}{(1+\bar{G})\left[(1+\epsilon) \theta U_{o}+\bar{G}\right]}$.

2. Similarly, although the value of $G$ associated with $U=U_{o}$ and $k=k_{o}$ does not satisfy the constraint $G \leq \bar{G}$, the binding constraint of $\bar{G}$ can be satisfied at $G=\sqrt{\theta U}$ by decreasing $U$. In this case, because decreasing $U$ decreases the value of $C$ at $G=\sqrt{\theta U}$ from the unconstrained maximum, its constrained maximum would occur with the constraint $G \leq \bar{G}$ just satisfied. The value of $G$ associated with $U=(1+\epsilon) \theta$ satisfies the constraint $G \leq \bar{G}$. Substituting $G=\bar{G}=\sqrt{\theta U}$ and equation (1) into the second row of equation (7), we find that, with the constraint $G \leq \bar{G}$ just satisfied, the local maximum for $C$ at $G=\sqrt{\theta U}$ is maximized either with $U=(1+\epsilon) \theta$ and $k=k_{o}$ or with $U=\bar{G}^{2} / \theta$ and $k=k_{o}$. The resulting value of $C$, denoted $\bar{C}^{I I}$, is either $\bar{C}^{I I}=\frac{[1+(1+\epsilon) \theta] \Omega_{o}-(1+\epsilon) \theta k_{o}}{(1+\theta \sqrt{1+\epsilon})^{2}}$ or $\bar{C}^{I I}=\frac{\left(1+\bar{G}^{2} / \theta\right) \Omega_{o}-\bar{G}^{2} k_{o} / \theta}{(1+\bar{G})^{2}}$.

3. The constraint $G \leq \bar{G}$ is not binding for the maximization of the local maximum for $C$ 
at $G=(1+\epsilon) \theta$. As in the previous section, the local maximum for $C$ at $G=(1+\epsilon) \theta$, if it exists, is maximized with $U=0$ and $C^{I I I}=\frac{\Omega_{\circ}}{1+(1+\epsilon) \theta}$.

We can easily confirm that $\bar{C}^{I}>\bar{C}^{I I}$ and $\bar{C}^{I}>C^{I I I}$, regardless of whether $\bar{C}^{I I}$ occurs with $U=(1+\epsilon) \theta$ or $U=\bar{G}^{2} / \theta$. Thus, $\bar{C}_{J}$ is associated with $U=U_{0}$ and $k$ and $G$ as given by equations (10) and (11). 


\section{References}

Alesina, Alberto, Baqir, Reza, and Easterly, William. "Public Goods and Ethnic Divisions", unpublished, March 1997.

Bourguignon, François and Verdier, Thierry. "Oligarchy, Democracy, Inequality, and Growth," unpublished, November 1966.

Brito, Dagobert and Intriligator, Michael. "Conflict, War, and Redistribution", American Political Science Review, 79, 1985, 943-957.

Easterly, William and Levine, Ross. "Africa's Growth Tragedy: Policies and Ethnic Divisions", Quarterly Journal of Economics, 112, November 1997, 1203-1250.

Grossman, Herschel I. "Production, Appropriation, and Land Reform," American Economic Review, 84, June 1994, 705-712.

Grossman, Herschel I. "Robin Hood and the Redistribution of Property Income," European Journal of Political Economy, 11, September 1995, 399-410.

Grossman, Herschel I. and Kim, Minseong. "Swords or Plowshares? A Theory of the Security of Claims to Property," Journal of Political Economy, 103, December 1995, 1275-1288.

Grossman, Herschel I. and Kim, Minseong. "Predation and Moral Decay," unpublished, April 1997a.

Grossman, Herschel I. and Kim, Minseong. "Predation, Efficiency, and Inequality," unpublished, December $1997 \mathrm{~b}$.

Usher, Dan. "Theft as a Paradigm for Departures from Efficiency," Oxford Economic Papers, 39, June 1987, 235-252; reprinted as Chapter III in Dan Usher, The Welfare Economics of Markets, Voting and Predation, Ann Arbor: The University of Michigan Press, 1992. 